\title{
Food and Age: It Takes Two to Degenerate
}

\author{
Raneen Nicola ${ }^{1,2}$ and Eitan Okun ${ }^{1,2,3 *}$ \\ ${ }^{1}$ The Leslie and Susan Gonda Multidisciplinary Brain Research Center, Bar-Ilan University, Ramat Gan, Israel, ${ }^{2}$ The Paul \\ Feder Laboratory on Alzheimer's Disease Research, Bar-Ilan University, Ramat Gan, Israel, ${ }^{3}$ The Mina and Everard Goodman \\ Faculty of Life Sciences, Bar-llan University, Ramat Gan, Israel
}

Keywords: aging, intermittent fasting, neurodegeneration, autophagy, Alzheimer's disease, caloric restriction

\section{A BLESSING AND A CURSE}

The twentieth century has brought numerous advances in technology, medicine, and food security. The reduced mortality of infants, toddlers, adults, and the elderly due to technological breakthroughs in medicine has brought a stable increase in the global expected lifespan at birth (Figure 1). Specifically, worldwide, the life expectancy of males rose from 59.6 years in the 1980's to 69.0 years in 2015, whereas the life expectancy of females increased from 63.7 to 74.8 years, respectively (Mortality and Causes of Death, 2016). This increase in lifespan is correlated with multiple age-dependent pathologies which have also increased in prevalence, such as neurodegenerative disorders (Hebert et al., 2013).

While food fermentation as a means to attain food security occurred as early as 5,000 years

OPEN ACCESS

Edited by:

Boon-Seng Wong,

Singapore Institute of

Technology, Singapore

Reviewed by:

Jetty Chung-Yung Lee,

The University of Hong Kong,

Hong Kong

Karthik Babu Mallilankaraman,

National University of

Singapore, Singapore

*Correspondence:

Eitan Okun

eitan.okun@biu.ac.il

Received: 03 April 2020

Accepted: 25 May 2020

Published: 26 June 2020

Citation:

Nicola R and Okun E (2020) Food and Age: It Takes Two to Degenerate. Front. Aging Neurosci. 12:182. doi: 10.3389/fnagi.2020.00182 ago, fermentation processes are believed to have been developed in order to preserve fruits and vegetables for times of scarcity in which food availability was intermittent (Medina-Pradas et al., 2017). This is not the situation nowadays, as food fermentation is not required for securing food anymore, but rather supplementing already available food stocks. The global increase in food security due to modern long-term food storage coupled with the increase in worldwide global food transportation, and international marketing has reduced the cost of food, increasing its availability in the developed world (Barnard, 2010) (Figure 1). However, food commercialization and the shift toward production of processed and ultra-processed foods have revealed clear adverse effects, such as the identification of processed food as a major cause for over-eating and the increase in the risk of metabolic syndrome, obesity, and diabetes (Hall et al., 2019). As the brain is one of the primary energy-demanding organs in the human body, it comes with no surprise that the brain is highly affected by such metabolic disorders as evident by recent epidemiological studies (Beydoun et al., 2008; Mule and Singh, 2018). For example, type-2 diabetes is strongly associated with cognitive impairment due to insulin resistance and altered glucose availability to neurons, which impair energy production capacity and proper neuronal function (Kandimalla et al., 2017).

Therefore, it's plausible to argue that the combined effect of the continued increase in lifespan and life-long continuous food consumption leads to a dramatic increase in the prevalence of neurodegenerative disorders in the elderly population. Herein, we will discuss factors that have shifted our nutritional habits over the last century. Next, we will delineate the effects of nutritional imbalance on neurodegenerative diseases at the cellular level, by shedding a light on the autophagy regulation. Moreover, we will discuss how Alzheimer's disease (AD), the most prevalent neurodegenerative disorder, estimated to affect 55 million Americans aged 65 and above, can be affected by such changes (Hebert et al., 2013; Brookmeyer et al., 2018). 


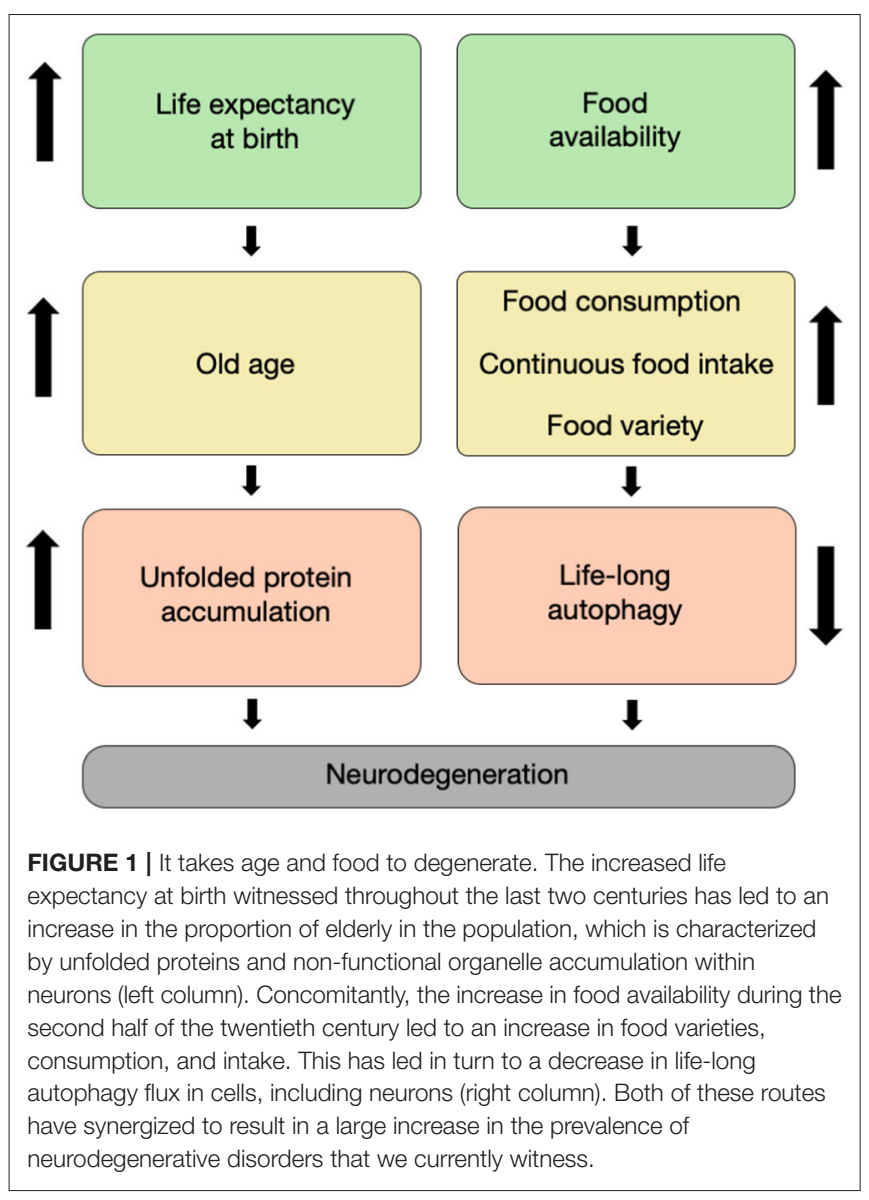

\section{A CENTURY OF MEDICAL REVOLUTIONS}

Early in the twentieth century, life expectancy at birth in most developed countries ranged between 45 and 50 years, with significant numbers of young children not reaching the age of 10 , mostly due to infectious diseases. Throughout the course of the last 60 years, however, the average lifespan at birth has risen linearly (Bell and Miller, 2005; Dong et al., 2016). Many factors can account for this, including early developments such as sanitation and clean water, which dramatically decreased infant mortality. Additional reasons include vaccine development against infectious agents, which significantly decreased children mortality from infections, the development of therapies for cardiovascular complications and cancer in older adults, and finally, the development of life-supporting devices for frail elderly people. As a result, over $95 \%$ of infants born in developed countries today will live to age 50 years or older, and over $84 \%$ of them will survive to age 65 years or older (Bell and Miller, 2005; Olshansky, 2018).

Although the maximal lifespan of humans has been shown by some mathematical models to be fixed (Dong et al., 2016), maximal lifespan at birth is still rising linearly, as for 160 years, the maximal life expectancy has witnessed consecutive increases by a quarter of a year per year (Oeppen and Vaupel, 2002).

\section{GLOBALIZATION AND FOOD SECURITY}

One of the outcomes of the industrial, technological and social changes in the twentieth century is the steep increase in the availability of food products, many of which are ready-made, and the consumption of foods of low-nutritional values and processed foods (Figure 1). Furthermore, it has been recently shown, in a well-controlled study in humans, that consumption of ultra-processed food [according to the NOVA system of food classification (Monteiro et al., 2018)] leads to increased caloric intake (Hall et al., 2019), which is thought to be at the basis of the obesity epidemic in the western world.

Children's food and beverage preferences and consumption are largely affected by media advertisements. Indeed, acute exposure to food advertising increases food intake specifically in children rather than in adults (Boyland et al., 2016), thus increasing the proportion of obese children who are at greater risk to develop type-2 diabetes at adulthood (Kelsey et al., 2014), which in itself is proposed to correlate with dementia (Biessels and Despa, 2018).

\section{EATING OURSELVES AWAY}

Aging is accompanied by a deterioration in multiple physiological aspects and can be characterized by cellular and molecular hallmarks, such as genomic instability, loss of proteostasis, cellular senescence, and more (López-Otín et al., 2013). This age-dependent decline in functioning also occurs in the brain, which becomes more vulnerable to oxidative stress, inflammatory insults, and metabolic stress. Moreover, multiple processes such as protein folding, degradation, and nutrientsensing may be damaged (Kemnitz et al., 1994; López-Otín et al., 2013; Cenini et al., 2019). This age-dependent decline in functioning is a major risk factor for several neurodegenerative diseases such as $\mathrm{AD}$, the most prevalent neurodegenerative disorder (Oddo, 2012; Cenini et al., 2019) (Figure 1). Another possible risk factor of neurodegenerative diseases is high caloric intake and obesity (Luchsinger et al., 2002; Beydoun et al., 2008). Accumulating evidence indicates that excessive food consumption may be harmful to the brain as continuous high glucose levels may increase oxidative stress, harming the vulnerable aging brain (Mule and Singh, 2018).

Studies in laboratory animals show that caloric restriction (decreased food intake or intermittent fasting) can extend lifespan in rodents and primates (Anderson et al., 2009; Colman et al., 2014) and delay the onset of age-related diseases such as hypertension and diabetes (Anderson et al., 2009; Colman et al., 2009; Fontana et al., 2010). Moreover, caloric restriction may protect neurons from degeneration and enhance adult neurogenesis and neuronal plasticity, which may protect the brain from a cognitive decline during aging and neurodegenerative diseases (Duan et al., 2001; Mattson et al., 2003) (Figure 1). It is still uncertain, however, how forms of caloric restriction or intermittent fasting affect Amyloidbeta $(A \beta)$ oligomerization and deposition and behavioral deficits in various $\mathrm{AD}$ rodent models. For example, both short- and long-term caloric restriction in APP/PS1 mice 
significantly decreased the accumulation of A $\beta$ (Patel et al., 2005; Mouton et al., 2009), while opposite data were shown when similar interventions were tested in the 5XFAD mouse model of AD (Lazic et al., 2020). Similar beneficial effects of caloric restriction were shown in animal models of Parkinson's Disease (PD) (Duan and Mattson, 1999; Maswood et al., 2004), providing evidence for a generalized role of reduced caloric intake in ameliorating neurodegeneration.

One of the crucial processes that are adversely affected during aging is cellular autophagy (Anderson et al., 2009; Rubinsztein et al., 2011), which is tasked with eliminating aggregated proteins, unhealthy organelles, and multiple intracellular components through their isolation in autophagosomes and fusion with lysosomes for breaking down these components ( $\mathrm{He}$ and Klionsky, 2009; Rubinsztein et al., 2011). This process allows the recycling of cellular macromolecules, which can be used to maintain energy and proper cell functioning (Kim and Guan, 2015). Accumulation of autophagic vesicles, which may contain undigested misfolded proteins, characterizes many neurodegenerative diseases, such as PD and AD (Lee et al., 2011).

Multiple mechanisms can explain the roles of fasting and caloric restriction in ameliorating neurodegeneration. One of the most studied mechanisms is the upregulation of autophagy via inhibiting mTOR activity, which promotes anabolic metabolism, necessary for protein synthesis and proliferation and cell growth, and inhibits catabolic activity (Kim and Guan, 2015). The mTOR pathway is activated by nutrient cues, such as amino acids, glucose (Gonzalez and Hall, 2017), and fat (Menon et al., 2017), which are abundant following food intake. Signaling events downstream to mTOR are involved in inhibition of cellular autophagy, a process that eliminates unfolded proteins and organelles within cells (Kim and Guan, 2015). This effect on mTOR activity could be mediated through two important energy sensors, namely, AMP-activated protein kinase (AMPK) and Sirtuin1 , and/or through decreasing downstream signaling by the insulin growth factor (IGF)-receptor (Rubinsztein et al., 2011). Thus, constant uptake of nutrients results in continuous activation of the mTOR pathway while concomitantly transcriptionally inhibiting the autophagy pathway, leading to life-long accumulation of unfolded proteins, a process that could promote neurodegeneration. These insights suggest that life-long enhancement of autophagy, whether by dietary or pharmacological means, can potentially prove vital to delaying the onset of neurodegenerative disorders in the elderly population.

While intermittent fasting and caloric restriction emphasize the effect of the timing of food consumption and food quantity on health and disease, other interventions, such as the ketogenic diet, focus instead on food quality. The concept of food quality vs. quantity in ketogenic diet cannot be dissociated, as ketogenic diet was shown to stimulate autophagy in the CNS (Mcdaniel et al., 2011) due to reduced circulating glucose and insulin levels (Paoli et al., 2014) while reducing mTOR activity (Mcdaniel et al., 2011). In the case of ketogenic diet, the elevated ketone bodies in the circulation are used as an alternative energetic metabolite to the brain, which is thought to be responsible for the beneficial effects of ketogenic diet in neurodegenerative disease such as AD (Kashiwaya et al., 2013; Wlodarek, 2019).

In the past decades, significant knowledge has accumulated regarding the importance of quantitative nutritional limitation of food consumption on maintaining proper homeostatic metabolism pathways, namely mTOR and autophagy, in various cell types and tissues. There is still a lack of deep understanding of the mechanisms regulated in the metabolically unique cellular environment of the brain, chiefly neurons, and astrocytes. Furthermore, although numerous studies have associated the consumption of certain types of nutrients with shortening or expanding lifespans in animals including primates (Mattison et al., 2017; Di Francesco et al., 2018; Wahl et al., 2018), the neurobiology community should move forward into studying how this applies to brain maintenance and function in young vs. aged animals. Moreover, causal, and not only associative links, between different food types and metabolic pathways in the brain should be studied. Lastly, we hope that new avenues of research into the impact of nutritional quantities and types on the aging human brain will be studied more rigorously in the near future, in order to provide the community with better tools for managing or even delaying the increasing rates of neurodegenerative diseases.

\section{CONCLUDING REMARKS AND FUTURE PERSPECTIVES}

The sobering statistics of one in three elderly people suffering from a type of age-related dementia call to devise a multipronged approach to targeting age-related neurodegenerative diseases. Synthesis of the current data indicates that not only age but also dietary lifestyles that changed dramatically during the twentieth century are at play. An expanding body of literature correlates dietary interventions with longevity. Indeed, many factors that are at play during aging have a role in promoting neurodegeneration, such as oxidative stress, accumulation of DNA damage, cell senescence, neuroinflammation, and decreased autophagic flux. Furthermore, most of these factors have both intrinsic and extrinsic drivers behind them. For example, aging, characterized by impaired sleep patterns (Mander et al., 2017), has been shown to mediate impaired DNA repair (Zada et al., 2019). Aging is also characterized by elevated levels of neuroinflammation that are transcriptionally regulated (Baruch et al., 2013). Autophagy, however, is a cellular pathway that throughout life is predominantly regulated extrinsically in a nutrient-consumption mediated manner. This places food consumption as a major factor, along with aging itself, in promoting neurodegenerative disorders. As one of the main aims of dietary regimes, such as intermittent fasting, is to inhibit mTOR and promote autophagy, it is yet unknown what the optimal timing is for this intervention in relation to the circadian rhythm. Furthermore, it is plausible that future research into mTOR inhibition by Rapamycin analogs, for example, can efficiently replace dietary interventions. 


\section{AUTHOR CONTRIBUTIONS}

EO and RN wrote the paper. All authors contributed to the article and approved the submitted version.

\section{REFERENCES}

Anderson, R. M., Shanmuganayagam, D., and Weindruch, R. (2009). Caloric restriction and aging: studies in mice and monkeys. Toxicol. Pathol. 37, 47-51. doi: 10.1177/0192623308329476

Barnard, N. D. (2010). Trends in food availability, 1909-2007. Am. J. Clin. Nutr. 91, 1530S-1536S. doi: 10.3945/ajcn.2010.28701G

Baruch, K., Ron-Harel, N., Gal, H., Deczkowska, A., Shifrut, E., Ndifon, W., et al. (2013). CNS-specific immunity at the choroid plexus shifts toward destructive Th2 inflammation in brain aging. Proc. Natl. Acad. Sci. U. S. A. 110, 2264-2269. doi: 10.1073/pnas.1211270110

Bell, F. C., and Miller, M. L. (2005). Life Tables for the United States Social Security Area, 1900-2100. Social Security Administration, Office of the Chief Actuary. Available online at: http://purl.access.gpo.gov/GPO/LPS80312

Beydoun, M. A., Beydoun, H. A., and Wang, Y. (2008). Obesity and central obesity as risk factors for incident dementia and its subtypes: a systematic review and meta-analysis. Obes. Rev. 9, 204-218. doi: 10.1111/j.1467-789X.2008.00473.x

Biessels, G. J., and Despa, F. (2018). Cognitive decline and dementia in diabetes mellitus: mechanisms and clinical implications. Nat. Rev. Endocrinol. 14, 591-604. doi: 10.1038/s41574-018-0048-7

Boyland, E. J., Nolan, S., Kelly, B., Tudur-Smith, C., Jones, A., Halford, J. C., et al. (2016). Advertising as a cue to consume: a systematic review and meta-analysis of the effects of acute exposure to unhealthy food and nonalcoholic beverage advertising on intake in children and adults. Am. J. Clin. Nutr. 103, 519-533. doi: 10.3945/ajcn.115.120022

Brookmeyer, R., Abdalla, N., Kawas, C. H., and Corrada, M. M. (2018). Forecasting the prevalence of preclinical and clinical Alzheimer's disease in the United States. Alzheimers Dement. 14, 121-129. doi: 10.1016/j.jalz.2017.10.009

Cenini, G., Lloret, A., and Cascella, R. (2019). Oxidative stress in neurodegenerative diseases: from a mitochondrial point of view. Oxid Med. Cell Longev. 2019:2105607. doi: 10.1155/2019/2105607

Colman, R. J., Anderson, R. M., Johnson, S. C., Kastman, E. K., Kosmatka, K. J., Beasley, T. M., et al. (2009). Caloric restriction delays disease onset and mortality in rhesus monkeys. Science 325, 201-204. doi: 10.1126/science.1173635

Colman, R. J., Beasley, T. M., Kemnitz, J. W., Johnson, S. C., Weindruch, R., and Anderson, R. M. (2014). Caloric restriction reduces age-related and all-cause mortality in rhesus monkeys. Nat. Commun. 5:3557. doi: 10.1038/ncomms4557

Di Francesco, A., Di Germanio, C., Bernier, M., and De Cabo, R. (2018). A time to fast. Science 362, 770-775. doi: 10.1126/science.aau2095

Dong, X., Milholland, B., and Vijg, J. (2016). Evidence for a limit to human lifespan. Nature 538, 257-259. doi: 10.1038/nature19793

Duan, W., Lee, J., Guo, Z., and Mattson, M. P. (2001). Dietary restriction stimulates BDNF production in the brain and thereby protects neurons against excitotoxic injury. J. Mol. Neurosci. 16, 1-12. doi: 10.1385/JMN:16:1:1

Duan, W., and Mattson, M. P. (1999). Dietary restriction and 2-deoxyglucose administration improve behavioral outcome and reduce degeneration of dopaminergic neurons in models of Parkinson's disease. J. Neurosci. Res. 57, 195-206. doi: 10.1002/(SICI)1097-4547(19990715)57:2<195::AID-JNR5>3.0.CO;2-P

Fontana, L., Partridge, L., and Longo, V. D. (2010). Extending healthy life spanfrom yeast to humans. Science 328, 321-326. doi: 10.1126/science.1172539

Gonzalez, A., and Hall, M. N. (2017). Nutrient sensing and TOR signaling in yeast and mammals. EMBO J. 36, 397-408. doi: 10.15252/embj.201696010

Hall, K. D., Ayuketah, A., Brychta, R., Cai, H., Cassimatis, T., Chen, K. Y., et al. (2019). Ultra-processed diets cause excess calorie intake and weight gain: an inpatient randomized controlled trial of ad libitum food intake. Cell Metab. 30, 67-77 e63. doi: 10.1016/j.cmet.2019.05.008

\section{FUNDING}

This work was funded by the Paul Feder Fund for Alzheimer's disease research.

He, C., and Klionsky, D. J. (2009). Regulation mechanisms and signaling pathways of autophagy. Annu. Rev. Genet. 43, 67-93. doi: 10.1146/annurev-genet-102808-114910

Hebert, L. E., Weuve, J., Scherr, P. A., and Evans, D. A. (2013). Alzheimer disease in the United States (2010-2050) estimated using the 2010 census. Neurology 80, 1778-1783. doi: 10.1212/WNL.0b013e31828726f5

Kandimalla, R., Thirumala, V., and Reddy, P. H. (2017). Is Alzheimer's disease a Type 3 diabetes? A critical appraisal. Biochim. Biophys. Acta Mol. Basis Dis. 1863, 1078-1089. doi: 10.1016/j.bbadis.2016.08.018

Kashiwaya, Y., Bergman, C., Lee, J. H., Wan, R., King, M. T., Mughal, M. R., et al. (2013). A ketone ester diet exhibits anxiolytic and cognitionsparing properties, and lessens amyloid and tau pathologies in a mouse model of Alzheimer's disease. Neurobiol. Aging 34, 1530-1539. doi: 10.1016/j.neurobiolaging.2012.11.023

Kelsey, M. M., Zaepfel, A., Bjornstad, P., and Nadeau, K. J. (2014). Age-related consequences of childhood obesity. Gerontology 60, 222-228. doi: $10.1159 / 000356023$

Kemnitz, J. W., Roecker, E. B., Weindruch, R., Elson, D. F., Baum, S. T., and Bergman, R. N. (1994). Dietary restriction increases insulin sensitivity and lowers blood glucose in rhesus monkeys. Am. J. Physiol. 266, E540-E547. doi: 10.1152/ajpendo.1994.266.4.E540

Kim, Y. C., and Guan, K. L. (2015). mTOR: a pharmacologic target for autophagy regulation. J. Clin. Invest. 125, 25-32. doi: 10.1172/JCI73939

Lazic, D., Tesic, V., Jovanovic, M., Brkic, M., Milanovic, D., Zlokovic, B. V., et al. (2020). Every-other-day feeding exacerbates inflammation and neuronal deficits in 5XFAD mouse model of Alzheimer's disease. Neurobiol. Dis. 136:104745. doi: 10.1016/j.nbd.2020.104745

Lee, S., Sato, Y., and Nixon, R. A. (2011). Lysosomal proteolysis inhibition selectively disrupts axonal transport of degradative organelles and causes an Alzheimer's-like axonal dystrophy. J. Neurosci. 31, 7817-7830. doi: 10.1523/JNEUROSCI.6412-10.2011

López-Otín, C., Blasco, M. A., Partridge, L., Serrano, M., and Kroemer, G. (2013). The hallmarks of aging. Cell 153, 1194-1217. doi: 10.1016/j.cell.2013.05.039

Luchsinger, J. A., Tang, M. X., Shea, S., and Mayeux, R. (2002). Caloric intake and the risk of Alzheimer disease. Arch. Neurol. 59, 1258-1263. doi: 10.1001/archneur.59.8.1258

Mander, B. A., Winer, J. R., and Walker, M. P. (2017). Sleep and human aging. Neuron 94, 19-36. doi: 10.1016/j.neuron.2017.02.004

Maswood, N., Young, J., Tilmont, E., Zhang, Z., Gash, D. M., Gerhardt, G. A., et al. (2004). Caloric restriction increases neurotrophic factor levels and attenuates neurochemical and behavioral deficits in a primate model of Parkinson's disease. Proc. Natl. Acad. Sci. U. S. A. 101, 18171-18176. doi: 10.1073/pnas.0405831102

Mattison, J. A., Colman, R. J., Beasley, T. M., Allison, D. B., Kemnitz, J. W., Roth, G. S., et al. (2017). Caloric restriction improves health and survival of rhesus monkeys. Nat. Commun. 8:14063. doi: 10.1038/ncomms14063

Mattson, M. P., Duan, W., and Guo, Z. (2003). Meal size and frequency affect neuronal plasticity and vulnerability to disease: cellular and molecular mechanisms. J. Neurochem. 84, 417-431. doi: 10.1046/j.1471-4159.2003.01586.x

Mcdaniel, S. S., Rensing, N. R., Thio, L. L., Yamada, K. A., and Wong, M. (2011). The ketogenic diet inhibits the mammalian target of rapamycin (mTOR) pathway. Epilepsia 52, e7-e11. doi: 10.1111/j.1528-1167.2011.02981.x

Medina-Pradas, E., Pérez-Díaz, I. M., Garrido-Fernández, A., and ArroyoLópez, F. N. (2017). "Review of vegetable fermentations with particular emphasis on processing modifications, microbial ecology, and spoilage," in The Microbiological Quality of Food: Foodborne Spoilers (Woodhead Publishing Series), 211-236. doi: 10.1016/B978-0-08-100502-6.00012-1 
Menon, D., Salloum, D., Bernfeld, E., Gorodetsky, E., Akselrod, A., Frias, M. A., et al. (2017). Lipid sensing by mTOR complexes via de novo synthesis of phosphatidic acid. J. Biol. Chem. 292, 6303-6311. doi: 10.1074/jbc.M116.772988

Monteiro, C. A., Cannon, G., Moubarac, J. C., Levy, R. B., Louzada, M. L. C., and Jaime, P. C. (2018). The UN Decade of Nutrition, the NOVA food classification and the trouble with ultra-processing. Public Health Nutr. 21, 5-17. doi: $10.1017 /$ S1368980017000234

Mortality, G. B. D., and Causes of Death, C. (2016). Global, regional, and national life expectancy, all-cause mortality, and cause-specific mortality for 249 causes of death, 1980-2015: a systematic analysis for the Global Burden of Disease Study 2015. Lancet 388, 1459-1544. doi: 10.1016/S0140-6736(16) 31012-1

Mouton, P. R., Chachich, M. E., Quigley, C., Spangler, E., and Ingram, D. K. (2009). Caloric restriction attenuates amyloid deposition in middle-aged dtg APP/PS1 mice. Neurosci. Lett. 464, 184-187. doi: 10.1016/j.neulet.2009. 08.038

Mule, N. K., and Singh, J. N. (2018). Diabetes mellitus to neurodegenerative disorders: is oxidative stress fueling the flame? CNS Neurol. Disord. Drug Targets 17, 644-653. doi: 10.2174/1871527317666180809092359

Oddo, S. (2012). The role of mTOR signaling in Alzheimer disease. Front. Biosci. 4, 941-952. doi: $10.2741 / \mathrm{s} 310$

Oeppen, J., and Vaupel, J. W. (2002). Demography. Broken limits to life expectancy. Science 296, 1029-1031. doi: 10.1126/science.1069675

Olshansky, S. J. (2018). From lifespan to healthspan. JAMA 320, 1323-1324. doi: 10.1001/jama.2018.12621

Paoli, A., Bianco, A., Damiani, E., and Bosco, G. (2014). Ketogenic diet in neuromuscular and neurodegenerative diseases. Biomed. Res. Int. 2014:474296. doi: $10.1155 / 2014 / 474296$
Patel, N. V., Gordon, M. N., Connor, K. E., Good, R. A., Engelman, R. W., Mason, J., et al. (2005). Caloric restriction attenuates Abetadeposition in Alzheimer transgenic models. Neurobiol. Aging 26, 995-1000. doi: 10.1016/j.neurobiolaging.2004.09.014

Rubinsztein, D. C., Mariño, G., and Kroemer, G. (2011). Autophagy and aging. Cell 146, 682-695. doi: 10.1016/j.cell.2011.07.030

Wahl, D., Solon-Biet, S. M., Wang, Q. P., Wali, J. A., Pulpitel, T., Clark, X., et al. (2018). Comparing the effects of low-protein and high-carbohydrate diets and caloric restriction on brain aging in mice. Cell Rep. 25, 2234-2243 e2236. doi: 10.1016/j.celrep.2018.10.070

Wlodarek, D. (2019). Role of ketogenic diets in neurodegenerative diseases (Alzheimer's disease and Parkinson's disease). Nutrients 11:169. doi: 10.3390/nu11010169

Zada, D., Bronshtein, I., Lerer-Goldshtein, T., Garini, Y., and Appelbaum, L. (2019). Sleep increases chromosome dynamics to enable reduction of accumulating DNA damage in single neurons. Nat. Commun. 10:895. doi: 10.1038/s41467-019-08806-w

Conflict of Interest: The authors declare that the research was conducted in the absence of any commercial or financial relationships that could be construed as a potential conflict of interest.

Copyright (c) 2020 Nicola and Okun. This is an open-access article distributed under the terms of the Creative Commons Attribution License (CC BY). The use, distribution or reproduction in other forums is permitted, provided the original author(s) and the copyright owner(s) are credited and that the original publication in this journal is cited, in accordance with accepted academic practice. No use, distribution or reproduction is permitted which does not comply with these terms. 